\title{
The immediate-shock deficit and postshock analgesia: Implications for the relationship between the analgesic $\mathrm{CR}$ and UR
}

\author{
MICHAEL S. FANSELOW, JESUS LANDEIRA-FERNANDEZ, JOSEPH P. DECOLA, \\ and JEANSOK J. KIM \\ University of California, Los Angeles, California
}

\begin{abstract}
Rats received a 3-sec, 1-mA footshock either immediately or 3 min after placement in a chamber. Postshock pain sensitivity was assessed with the formalin test. The animals that received the 3-min delay between placement and shock showed an analgesic response compared with noshock controls. The immediate-shock animals did not. Thus the immediate-shock deficit, previously reported for freezing and defecation, also occurs for analgesia. This suggests that shock levels sufficient to condition analgesia are not necessarily sufficient to produce analgesia as an unconditional response. As with freezing, there is a dissociation between conditional and unconditional responses in the fear-conditioning system. Increasing immediate-shock levels to 6 sec, $2 \mathrm{~mA}$ produced a transient unconditional analgesia. For analgesia, a conditional response is more readily produced than an unconditional response.
\end{abstract}

An interesting aspect of fear-conditioning situations is that unconditional responses (UR) and conditional responses (CR) appear to be strikingly different. For example, rats' immediate UR to an aversive electric shock is vigorous locomotor behavior called the activity burst (Fanselow, 1982). However, stimuli paired with shock elicit a pronounced immobility termed freezing (Fanselow, 1980). Another example is the reduction in pain sensitivity, or analgesia, that occurs with fear-conditioning procedures (Watkins, Cobelli, \& Mayer, 1982). Following electric shock to their hind feet, rats show an analgesia that is not affected by opioid antagonists. Yet, stimuli associated with hind-foot shock produce a conditional analgesia eliminated by opioid antagonists. Thus, for analgesia, although the CR and UR are superficially the same, the mediating mechanisms differ substantially.

This divergence of CR and UR in fear conditioning has fostered considerable discussion, because it is contrary to traditional stimulus substitution theory (e.g., Fanselow, 1989). If one observes a rat after unsignaled shock, its immediate reaction is an activity burst, but as this reaction fades the rat begins to freeze (e.g., Bolles \& Riley, 1973). On the basis of this pattern, Mackintosh (1974)

This research was supported by National Institute of Mental Health Grant MH39786 to M.S.F. This article was prepared while the first author was a fellow at the Center for Advanced Study in the Behavioral Sciences, supported by John D. and Catherine T. MacArthur Foundation Grant 8900078 . J.L.-F. was supported by a scholarship from Consehlo Nacional de Desenvolvimento Cientifico of the Ministry of Science, Brazil. J.J.K. is now at the Neuroscience Program, University of Southern California. Address reprint requests to the first author at Department of Psychology, University of California, Los Angeles, CA 90024-1563. argued that freezing may be a delayed UR to shock, and it is this delayed UR that becomes conditioned. More recently, Wagner and Brandon (1989) restated this view in a somewhat more formal manner. According to Wagner's (1981) SOP model, upon its presentation, an unconditional stimulus (US) is represented in memory in a very active form, called A1. With time, the memorial state of this representation eventually degrades to a less active state, termed A2, before it becomes inactive. Explicit in Wagner's theorizing is the assumption that a conditional stimulus (CS) can activate a memorial representation of the US, but only into the A2 state. Wagner suggests that the $\mathrm{A} 1$ and $\mathrm{A} 2$ states may be linked to different behaviors; this aspect of the theory allows it to handle divergences of the CR and UR. For example, Wagner and Brandon suggest that the activity burst to electric shock might be generated by the $A 1$ state and that freezing is generated by the $A 2$ state. The immediate reaction to shock is an activity burst that persists as long as shock is represented in the A1 state. As the memory degrades from $\mathrm{A} 1$ to $\mathrm{A} 2$, freezing begins to be observed, hence the frequently reported delayed recruitment of postshock freezing (e.g., Fanselow \& Bolles, 1979). Because a CS only activates the shock memory into the $A 2$ state, the CR is freezing without an activity burst. Grau (1987) has applied the SOP principles to analgesia, linking opioid-antagonist-insensitive analgesia to the A1 state and opioid-antagonist-sensitive analgesia to the A2 state. Thus, Grau (1987) suggests that because a CS can only activate the memory of shock into the A2 state, conditional analgesia is always opioid antagonist sensitive, even when the immediate UR to shock is an antagonist-insensitive analgesia.

We have offered an alternative formulation (e.g., Fanselow, 1991; Fanselow, DeCola, \& Young, 1993) that 
suggests that different, often competing, neural mechanisms mediate the CR and UR. According to this view, conditional fear responses, such as freezing, are generated by a CS's ability to activate a defensive behavior system mediated by the amygdala and ventral portions of the periaqueductal gray (PAG). On the other hand, unconditional responses, such as the activity burst, are mediated by the shock's ability to directly activate the dorsal-lateral portions of the PAG. A basic assumption of this defensive behavior system view is that CR and UR should be entirely separable and conditional fear responses need not be mimics of the UR, in any sense. According to this approach, the delayed freezing seen following unsignaled shock is a CR to contextual stimuli that are present both before and after shock.

One way of testing the SOP and the defensive behavior system alternatives is to present a US in such a way that it provokes a UR without provoking a CR. Such a procedure has been examined for freezing (Fanselow, 1986, 1990). A rat shocked immediately upon placement in a context does not freeze following shock. Indeed, the overt activity of such animals is not behaviorally distinguishable from unshocked controls. In this immediate-shock preparation, the animal has inadequate time to process the contextual CS's before shock, so a conditional response is precluded. However, the animals receive the full unconditional stimulus and, therefore, a UR should be intact. Recently, Kiernan and Cranney (1992) provided an important demonstration of this point. They reported that the unconditional startle to a loud noise was equivalent, whether the stimulus was presented immediately or $1 \mathrm{~min}$ after placement in an apparatus. However, freezing occurred only in the delayed US condition. Thus, the results with immediate US delivery suggest that freezing is not at all an unconditional response to the US (converging lines of evidence on this point are reviewed elsewhere, e.g., Fanselow, 1989).

This immediate-shock deficit indicates that freezing is exclusively a CR to shock-associated cues and not a UR to the shock itself. An analysis of the analgesia that follows shock has not been conducted, which was the purpose of the present study. The basic question was whether the immediate-shock deficit described for freezing also occurs with the analgesia that is a typical CR in fearconditioning preparations. The formalin test of pain sensitivity served as our assay of analgesia. This test has been used extensively to document fear-induced analgesia (e.g., Fanselow \& Baackes, 1982; Fanselow, Calcagnetti, \& Helmstetter, 1989). It allows pain sensitivity to be monitored in unrestrained animals. Using restrained animals in an immediate-shock procedure would be logistically difficult.

\section{EXPERIMENT 1}

\section{Method}

Subjects. The subjects were 32 male rats of Long-Evans descent, bred in the psychology department of the University of California, Los Angeles. They were about 100 days old at the start of the experiment. The rats, maintained on a 12:12-h light:dark cycle, were group housed until 5 days before the experiment. Then they were housed individually and adapted to handling and transport to the laboratory during those 5 days. The animals always had ad-lib access to food and water.

Apparatus. The apparatus consisted of four typical observation chambers $(28 \times 21 \times 10.5 \mathrm{~cm}$; Lafayette Instrument $\mathrm{Co}$., North Lafayette, IN), which were in an isolated room. They were placed inside sound-attenuation chests that had their front doors removed to facilitate observation. Two ceiling-mounted fluorescent bulbs illuminated the room. The floors consisted of $184-\mathrm{mm}$-diam stainless steel rods spaced $1.5 \mathrm{~cm}$ apart (center to center). After each use, the chamber was carefully cleaned with a 5\% ammonium hydroxide solution. Shock was scrambled by a Lafayette Instrument Co. mechanical scrambler.

A video camera, mounted on a wall in the isolated experimental room, was connected to a video monitor in a separate observation room. This allowed observation of the animals without disturbing them.

Procedure. The rats were injected with $.05 \mathrm{ml}$ of a $7.5 \%$ formalin and distilled water solution, subcutaneously, into the ventral surface of the left hind foot. Thirty minutes later, they were placed into the chambers, because formalin-induced behaviors reach a stable maximum during this period (Dubuisson \& Dennis, 1977). Four rats were run at a time. Immediate-shock rats $(n=10)$ received a 1-mA, 3-sec shock as soon as the doors of the observation chamber were closed. Observation began when shock terminated. The immediate-control group $(n=6)$ received the same treatment without shock. Delayed-shock $(n=10)$ rats were placed into the chamber and waited $3 \mathrm{~min}$ for shock. Again, observation started at shock termination. Delay-control rats $(n=6)$ were treated like the delayed-shock animals, but no shock was given. The two types of no-shock controls were run because the onset of observation times for the two groups had to differ if observation was to start immediately after shock.

Two experimenters handled the rats and operated the equipment; a third observed. This observer was not informed of the subject's treatment condition and did not enter the observation room until the shock had ended. The observation period was $8 \mathrm{~min} 32 \mathrm{sec}$ following shock; a comparable period was used for the two no-shock control groups. The observer used a time-sampling procedure: A pacing tone sounded every $2 \mathrm{sec}$ and 1 animal's behavior was scored according to what it was doing at that instant. Each animal was scored sequentially, so observation of a particular subject occurred once every $8 \mathrm{sec}$. Behavior was judged as formalin-induced recuperative behavior (see Fanselow, 1984, for photographs) or other behavior. Recuperative behavior consisted of either raising the injected foot and holding it close to the body or licking the injected foot. A suppression of this pain-related behavior indicates analgesia. Later, a blind observer videotaped and analyzed the behavior for freezing. The same time-sampling procedure was used, except that the rats were scored as either freezing, defined as the absence of movements, including sniffing, or not. Only the minimal movement of the flanks necessary for respiration is tolerated in our definition of freezing.

\section{Results}

As in past analyses with the formalin test, the percentage of samples scored as recuperative behavior was determined for each animal, and these scores were subjected to an analysis of variance followed by planned pairwise comparisons. For all contrasts, the significance criterion was $p<.05$.

The group means, shown in Figure 1, were reliably different $[F(3,28)=5.95]$. The immediate-control animals showed a trend toward less recuperative behavior than the delay controls, although this difference fell just short of statistical reliability $[F(1,28)=3.34]$. Apparently, the 
timing of placement in the chamber has some influence on formalin-treated rats in the absence of shock. Because of this trend, the two shocked groups were only compared with their placement controls, and not with a pooled noshock control.

Delayed shock virtually eliminated recuperative behavior $[F(1,28)=11.64]$. On the other hand, immediate shock tended to increase the amount of recuperative behavior, although this trend was not statistically reliable $[F(1,28)=3.15]$.

It is possible that immediate shock may have produced a brief analgesia that was not detected in the overall percentage of recuperative behavior over the 8 -min period. Therefore, a supplemental analysis was undertaken on the latency to the first sample scored as recuperation, and this indicated a reliable group difference $[F(3,28)=$ 8.85]. As shown in Figure 2, the two controls differed $[F(1,28)=51.63]$, so the shock groups were only contrasted with their respective no-shock controls. There was an increased latency to recuperate in the delayed-shock animals compared with the delay controls $[F(1,28)=$ 195.26]. The immediate-shock animals did not differ reliably from their controls $[F(1,28)=3.73]$, but the trend was toward a decreased latency to react to the formalin treatment.

Observation of the videotapes indicated that the only rats that froze were the ones that had received delayed shock $(M=19 \%$ of the samples, $S E M=5.67)$. None of the rats in either the immediate-shock or the two noshock control groups ever displayed any instances of freezing. Therefore, immediate shock resulted in a complete deficit in freezing.

\section{Discussion}

When the rats received a single shock after being in a chamber for $3 \mathrm{~min}$, they were analgesic following shock.

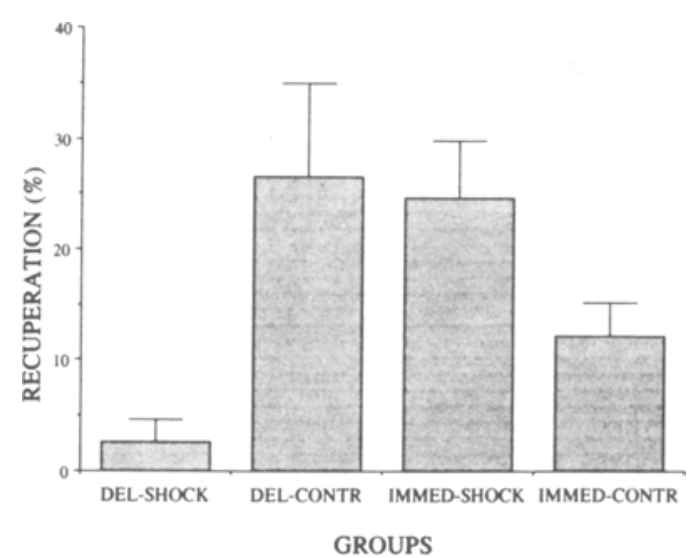

Figure 1. The mean (and SEM) percentage of observations indicating the rats in Experiment 1 in formalin-induced recuperative behavior. DEL indicates groups that had shock 3 min after placement in the observation chamber. IMMED refers to groups given shock immediately after placement in the chamber. SHOCK indicates groups that received a 1-mA, 3-sec shock. CONTR refers to groups that received no shock, but were observed at time intervals that corresponded to their respective shock conditions.

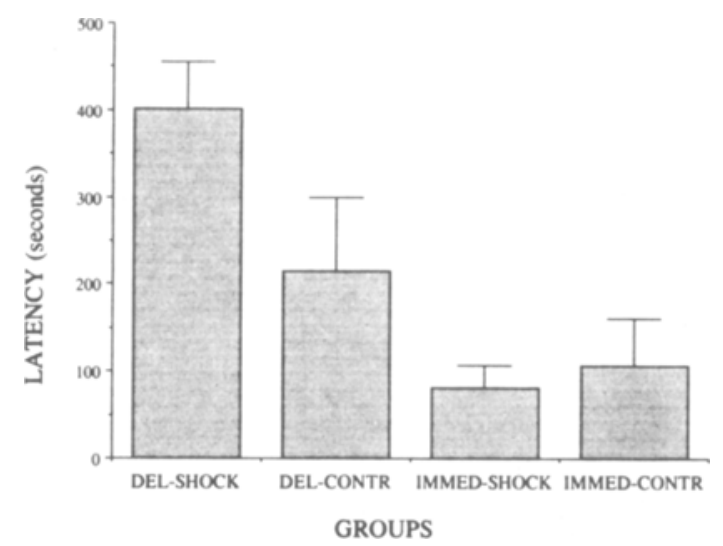

Figure 2. The mean (and SEM) latency, in seconds, until the first incidence of formalin-induced recuperative behavior was scored in Experiment 1. DEL indicates delayed-shock conditions, and IMMED indicates immediate-shock conditions. CONTR rats are unshocked controls.

This analgesia was indicated by a general suppression of formalin-induced recuperative behavior over the 8 -min test period and an increased latency to react to a painful formalin injection. There was not even a hint that any such analgesia occurred when the same shock was given immediately upon placement into the chamber. As in previous studies (Fanselow, 1986, 1990), immediate shock also did not condition freezing.

The lack of analgesia in the immediate-shock group suggests that these shock parameters do not generate analgesia as a UR. This implies that the analgesia found after delayed shock was purely a conditional response. Further evidence toward this conclusion is that analgesia is reduced by a context shift between training and testing, but not by a temporal delay between training and testing (Fanselow, 1984). Under these conditions, a shock that fails to produce either freezing or analgesia as a UR is successful in conditioning both of these responses.

\section{EXPERIMENT 2}

One premise of the original perceptual-defensiverecuperative model of Bolles and Fanselow (1980) is that the analgesia following shock is always a conditional reaction. The present data are consistent with that theory. However, several laboratories have provided evidence that strongly suggests that, under some conditions, analgesia can be unconditionally elicited by shock. For example, analgesia occurs in deeply sedated rats (e.g., Maier, 1989; Terman, Shavit, Lewis, Cannon, \& Liebeskind, 1984) and some analgesia survives a CS preexposure treatment (Maier \& Watkins, 1991). Most of the experiments that have demonstrated an unconditional analgesia to shock have presented more shock than that used here and in typical fear-conditioning experiments. For example, Terman et al. (1984) found analgesia in sedated animals after $30 \mathrm{sec}$ of $2.5-\mathrm{mA}$ footshock. Thus, it may be that unconditional analgesia requires more severe shock parameters 


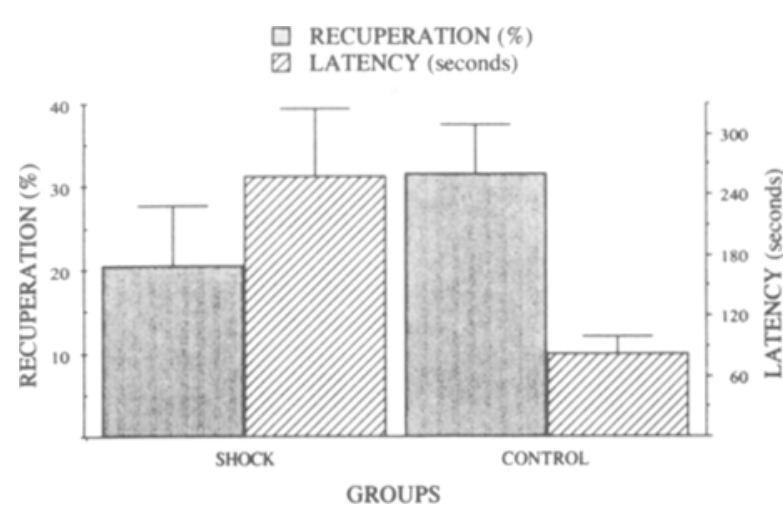

Figure 3. The means (and SEMs) for the SHOCK $(2 \mathrm{~mA}, 6 \mathrm{sec})$ and CONTROL (unshocked) groups of Experiment 2. The percentage of the observation period in which the rats engaged in recuperative behavior is indicated by the shaded bars (axis to the left) and the latency to the first incidence of recuperation is indicated by the striped bars (axis to the right).

than those used in Experiment 1. The purpose of Experiment 2 was to determine whether increasing the impact of the shock would result in an analgesia with immediate shock delivery.

\section{Method}

Two groups of 8 animals, similar to those in the first experiment, were used. The apparatus was the same. The procedure replicated the two "immediate"' groups of the first experiment; however, the immediate shock was a 2-mA, 6-sec shock.

\section{Results and Discussion}

There was no overall difference in the percentage of recuperative behavior during the 8 -min test $[F(1,14)=$ 1.36]. However, in contrast to the results of Experiment 1 , the trend was toward less recuperative behavior in the immediately shocked rats when averaged over the entire test period (see Figure 3). The latency data seemed to be a more sensitive measure than overall recuperative behavior for gauging possible unconditional analgesic reaction, because the immediate-shock rats took three times as long to initiate recuperative behavior. This reliable increase in latency to react to the formalin $[F(1,14)=$ 6.29] suggests that immediate shock did produce a brief unconditional analgesia when both intensity and duration were doubled from that used in Experiment 1. The greater sensitivity of the latency measure suggests that this unconditional analgesia is very brief.

Because of a faulty videotape, the data on freezing were lost. However, unpublished research from our laboratory using similar parameters has confirmed that the immediateshock deficit is as pronounced as that reported for the first experiment.

\section{GENERAL DISCUSSION}

A single 1-mA, 3-sec shock was followed by analgesia when rats were placed in the shock context 3 min before shock. This analgesia manifested itself in both the latency to react to a formalin injection and the total amount of formalin-related behavior observed. The analgesia following delayed shock appears to be a conditional reaction, because the same shock given immediately after placement into the chamber did not produce this reaction. These data suggest that a shock sufficient to condition analgesia is not necessarily sufficient to produce analgesia as an unconditional reaction. Such data suggest that the mechanisms responsible for conditioning and generating unconditional responses are quite distinct; the CR does not mimic the UR. Note that the CR is frequently weaker than the UR (e.g., Mackintosh, 1974). The present data demonstrate the opposite outcome; the analgesic CR was stronger than the UR. For fear conditioning, these analgesia data, along with data on freezing (e.g., Fanselow, 1986,1990 ), call into question the applicability of any model that requires the $C R$ to be any sort of mimic of the UR, whether the UR is an immediate or delayed reaction to the US (e.g., Grau, 1987; Wagner \& Brandon, 1989).

If rats are removed from a delayed-shock context after the shock and placed in a different context, freezing and analgesia can be eliminated even if the test in the new context occurs right after the shock (D. C. Blanchard \& R. J. Blanchard, 1969; Fanselow, 1981, 1984). The loss of freezing and analgesia following immediate shock complements the earlier results with context shift designs. Therefore, the context shift and immediate-shock procedures provide converging lines of evidence that these reactions can be conditioned by the US, even when they are not unconditionally provoked by the US. This pattern of findings is inconsistent with models, such as the SOP, that suggest that CRs should resemble, at the very least, delayed URs (Grau, 1987; Wagner \& Brandon, 1989). However, context shift designs are open to an alternative interpretation that is consistent with the SOP model: Rats must be handled to move them to the different context. Usually, both the shifted and unshifted controls are handled equivalently. This movement may act as a distractor that, according to the SOP model, accelerates memorial decay (Wagner \& Brandon, 1989). Context shifts may eliminate postshock responding because of a distraction effect on the UR, rather than elimination of the conditional cues that support the CR (Grau, 1987). Given this interpretation of context shift preparations, the immediateshock procedure becomes especially problematic for those models because the animals are undisturbed between delivery of the US and the test. Without such a disturbance, it becomes difficult to postulate that distracting variables between shock and testing cause a disruption of the UR.

Analgesia following immediate shock was observed when the intensity and duration of shock were doubled. This pattern is generally consistent with the literature on shock-induced analgesia (e.g., Fanselow, 1984; Terman et al., 1984). Researchers who found that analgesia was purely a CR tended to use milder shock parameters than those who provided a strong suggestion of a UR. For example, Maier (1989) reported that the duration of the UR 
to shock increased with repeated exposures to 5-sec shocks.

The data of Experiment 2 are not consistent with the strong statement of the original perceptual-defensiverecuperative model that analgesia following shock is always a CR and never a UR (Bolles \& Fanselow, 1980). However, they are consistent with more current versions of that model (e.g., Fanselow, 1991). According to this view, the fear $C R$ is learned activation of a defensive behavior system module that is normally activated by moderate degrees of threat. In a natural situation, an example of a moderate threat would be detection of a predator in the vicinity; the ventral PAG organizes that defensive behavior module. Intense threats, such as a predator's making physical contact, activate a different defensive behavior module organized by the dorsal-lateral PAG. It is this system that shock unconditionally activates. Obviously, more intense stimuli would be necessary to activate the latter module. It is important that, although direct stimulation of either the ventral or dorsal-lateral PAG can produce analgesia, these analgesias are distinct in their forms (Cannon, Prieto, Lee, \& Liebeskind, 1982).

The original explanation of the immediate-shock deficit in freezing was that immediate-shocked rats did not freeze-not because of a deficit in fear, but because they were searching for an escape exit (R. J. Blanchard, Fukunaga, \& D. C. Blanchard, 1976). According to this performance account, delayed-shock animals froze because they learned, during the preshock period, that there were no escape exits. If this explanation is correct, then analgesia would still be expected in the immediate-shock animals. Thus, the present findings add to the body of evidence indicating that the immediate-shock deficit arises from a failure to associate the context with shock rather than a specific failure to perform the freezing response (Fanselow, 1986, 1990; Fanselow et al., 1993).

\section{REFERENCES}

Blanchard, D. C., \& Blanchard, R. J. (1969). Crouching as an index of fear. Journal of Comparative \& Physiological Psychology, 67, 370-375.

Blanchard, R. J., Fukunaga, K. K., \& Blanchard, D. C. (1976). Environmental control of defensive reactions to footshock. Bulletin of the Psychonomic Society, 8, 129-130.

Bolles, R. C., \& Fanselow, M. S. (1980). A perceptual-defensiverecuperative model of fear and pain. Behavioral \& Brain Sciences, 3, 291-301.

Bolles, R. C., \& Riley, A. L. (1973). Freezing as an avoidance response: Another look at the operant-respondent distinction. Learning \& Motivation, 4, 268-275.

Cannon, J. T., Prieto, G. J., Lee, A., \& Liebeskind, J. C. (1982). Evidence for opioid and non-opioid forms of stimulation-produced analgesia in the rat. Brain Research, 243, 315-321.

Dubuisson, D., \& Dennis, S. G. (1977). The formalin test: A quantitative study of the analgesic effects of morphine, meperidine, and brain stimulation in rats and cats. Pain, 4, 161-174.

FANSELow, M. S. (1980). Conditional and unconditional components of post-shock freezing in rats. Pavlovian Journal of Biological Sciences, 15, 177-182.

Fanselow, M. S. (1981). Naloxone and Pavlovian fear conditioning. Learning \& Motivation, 12, 398-419.
FANSELOW, M. S. (1982). The postshock activity burst. Animal Learning \& Behavior, 10, 448-454.

FanSELOW, M. S. (1984). Shock-induced analgesia on the formalin test: Effects of shock severity, naloxone, hypophysectomy, and associative variables. Behavioral Neuroscience, 98, 79-95.

FANSElow, M. S. (1986). Associative vs. topographical accounts of the immediate shock-freezing deficit in rats: Implications for the response selection rules governing species-specific defensive reactions. Learning \& Motivation, 17, 16-39.

FANSELOW, M. S. (1989). The adaptive function of conditioned defensive behavior: An ecological approach to Pavlovian stimulus substitution theory. In R. J. Blanchard, P. F. Brain, D. C. Blanchard, \& S. Parmigiani (Eds.), Ethoexperimental approaches to the study of behavior (NATO ASI Series D, Vol. 48, pp. 151-166). Boston: Kluver Academic Publishers.

FANSELOW, M. S. (1990). Factors governing one-trial contextual conditioning. Animal Learning \& Behavior, 18, 264-270.

FANSELOW, M. S. (1991). The midbrain periaqueductal gray as a coordinator of action in response to fear and anxiety. In A. Depaulis \& R. Bandler (Eds.), The midbrain periaqueductal gray matter: Functional, anatomical and immunohistochemical organization (pp. 151173). New York: Plenum.

Fanselow, M. S., \& BaAckes, M. P. (1982). Conditioned fear-induced opiate analgesia on the formalin test: Evidence for two aversive motivational systems. Learning \& Motivation, 13, 200-221.

FANSELOW, M. S., \& BolLES, R. C. (1979). Naloxone and shock-elicited freezing in the rat. Journal of Comparative \& Physiological Psychology, 93, 736-744.

Fanselow, M. S., Calcagnetti, D. J., \& Helmstetter, F. J. (1989). The role of $\mu$ and $x$ opioid receptors in conditional-fear induced analgesia: The antagonistic actions of nor-binaltorphimine and the cyclic somatostatin octapeptide, cys $^{2}$ tyr $^{3} \mathrm{orn}^{5} \mathrm{pen}^{7}$-amide. Journal of Pharmacology \& Experimental Therapeutics, 250, 825-830.

Fanselow, M. S., DeCola, J. P., \& Young, S. L. (1993). Mechanisms responsible for reduced conditioning with massed unsignaled unconditional stimuli. Journal of Experimental Psychology: Animal Behavior Processes, 18, 121-137.

GRAU, J. W. (1987). The central representation of an aversive event maintains the opioid and nonopioid forms of analgesia. Behavioral Neuroscience, 101, 272-288.

Kiernan, M., \& Cranney, J. (1992). Immediate-startle stimulus presentation fails to condition freezing responses to contextual cues. $\mathrm{Be}$ havioral Neuroscience, 106, 121-124.

Mackintosh, N. J. (1974). The psychology of animal learning. New York: Academic Press.

MAIER, S. F. (1989). Determinants of the nature of environmentally induced hypoalgesia. Behavioral Neuroscience, 103, 131-143.

Maier, S. F., \& WATkins, L. R. (1991). Conditioned and unconditioned stress-induced analgesia: Stimulus preexposure and stimulus change. Animal Learning \& Behavior, 19, 295-304.

Terman, G. W., Shavit, Y., Lewis, J. W., Cannon, J. T., \& LieBESKIND, J. C. (1984). Intrinsic mechanisms of pain inhibition: Activation by stress. Science, 226, 1270-1277.

WAGNER, A. R. (1981). SOP: A model of automatic memory processing in animal behavior. In N. E. Spear \& R. R. Miller (Eds.), Information processing in animals: Memory mechanisms (pp. 5-47). Hillsdale, NJ: Erlbaum.

WAGNER, A. R., \& Brandon, S. E. (1989). Evolution of a structured connectionist model of Pavlovian conditioning. In S. B. Klein \& R. R. Mowrer (Eds.), Contemporary learning theories: Pavlovian conditioning and the status of traditional learning theory (pp. 149-189). Hillsdale, NJ: Erlbaum.

WATkins, L. R., Cobelli, D. A., \& MAYer, D. J. (1982). Classical conditioning of front paw and hind paw footshock induced analgesia (FSIA): Naloxone reversibility and descending pathways. Brain $R e$ search, 243, 119-132.

(Manuscript received March 2, 1993; revision accepted for publication June 27, 1993.) 